\title{
Postlaminectomy Bone and Scar Formations in Presence of Ankaferd Blood Stopper and Bitter Melon (Momordica Charantia): An Experimental Study
}

\author{
Enis KURUOGLU1', Mehmet Emin ONGER ${ }^{2}$, Abdullah Hilmi MARANGOZ¹, Suleyman Emre KOCACAN², \\ Cengiz COKLUK ${ }^{1}$, Suleyman KAPLAN² \\ 1Ondokuz Mayıs University, Medical Faculty, Department of Neurosurgery, Samsun, Turkey \\ ${ }^{2}$ Ondokuz Mayıs University, Medical Faculty, Department of Histology and Embryology, Samsun, Turkey \\ ${ }^{3}$ Ondokuz Mayıs University, Medical Faculty, Department of Physiology, Samsun, Turkey
}

\section{ABSTRACT}

AIM: A quantitative model of postlaminectomy was designed in rats. The effects of Momordica Charantia (MC) and Ankaferd blood stopper (ABS) on the bone and scar formation after laminectomy were concurrently evaluated.

MATERIAL and METHODS: Eighteen adult Wistar albino rats underwent lumbar laminectomy at L2-L3 vertebral levels, and were randomly assigned to one of three groups of six rats each. The Treatment group received MC and ABS treatment and the Control group was left untreated. Rats were sacrificed 4 weeks after treatment. Then; the lumbar spine was excised en-block, fixed and decalcified. Sections were stained with hematoxylin and eosin (H\&E) and Masson's trichrome, and evaluated for peridural fibrosis (PF), new bone formation, and vascular proliferation.

RESULTS: Total volume of new bone in the MC group was significantly increased in comparison to the Control group ( $p<0.05)$. Also; there was highly significant increase in terms of the total volume of fibrous tissue in the MC and ABS groups when compared with the Control group $(p<0.01)$. Besides; there was a highly significant difference between the $M C$ and the Control groups ( $<<0.01)$ in point of total volume of vessel.

CONCLUSION: Both MC and ABS are not convenient to prevent the PF formation and MC may promote new bone formation and angiogenesis after lumbar laminectomy in rats.

KEYWORDS: Bone formation, Fibrosis, Histology, Laminectomy, Rat

\section{INTRODUCTION}

$\mathrm{P}$ eridural fibrosis (PF) is an undesirable outcome of decompressive spinal surgery that may compress the dura and nerve roots (3). PF develops out of the laminectomy membrane, which covers the defect created by the bone resection to replace the resected areas. Commonly, the bone defects as a result of laminectomy regenerate as fibrous tissue that intends to regenerate resected lamina (24). Briefly; $\mathrm{PF}$ is a part of the natural healing process and it could be observed after laminectomy and revealed by magnetic resonance imaging (MRI) (22). Recently, more attention has been focused on PF, which may be responsible for recurrent pain after laminectomy $(9,16)$ to prevent post laminectomy scarring and to induce new bone formation in different approaches $(5,8,30)$. A number of synthetic and natural materials have been used to prevent or reduce PF and induce new bone formation after laminectomy in both animal and human studies (22). Especially, the PASS principles (Primary wound closure/ 
Angiogenesis/Space/Stability) for satisfactory bone regeneration must be addressed for a successful spinal fusion (29).

In developing countries, $80 \%$ of the population continues to use traditional medicine in primary medical problems. In the past decade, therefore, research has been focused on scientific evaluation of traditional drugs of plant origin. At this point; $\mathrm{MC}$ is one of the plants that have been frequently used as a medicine (11). MC, which belongs to the family Cucurbitaceae, is a tropical spreading plant and is commonly known by the bitter taste (13). The plant has 228 different compounds, which help medicinally either alone or when combined (19). Therefore, people drink the pulp of the fruit as a juice or tea and eat the fruit raw as a vegetable as a traditional herb for treating various diseases such as fewer, common cold, rheumatism and wounds $(20,26,31)$.

Another antioxidant, ABS is a folkloric medicinal plant extract, which is used as a haemostatic agent, and it has been used as a blood-stopping agent against various types of bleeding (10). It consists of 5 different plant extracts: Urtica dioica, Vitis vinifera, Glycyrrhiza glabra, Alpinia officinarum, and Thymus vulgaris. Studies have shown that ABS plays important roles in several metabolic pathways involved in erythrocyte aggregation, tissue oxygenation and physiological processes, etc. (27). Besides its bleeding control properties, ABS reportedly accelerates early bone healing and formation by affecting cell proliferation and cell mediators $(4,10)$. Therefore, it is thought to trigger new pathways in the application area (27).

The aim of our study was to investigate the effects of ABS and $\mathrm{MC}$ on the formation of PF and osteogenesis in a rat postlaminectomy model.

\section{- MATERIAL and METHODS}

Eighteen adult Wistar albino rats weighting 200-250 g were used in this study. The experimental animal protocol was carried out at Ondokuz Mayıs University School of Medicine with approval from the local ethic commission.

For the purpose of preventing prophylaxis, a single dose of $50 \mathrm{mg} / \mathrm{kg}$ ceftriaxone (Rocephine, Roche, Türkiye) was administered intraperitoneally 30 minutes before the operation. Animals were anesthetized with intramuscular $50 \mathrm{mg} / \mathrm{kg}$ ketamine (Ketalar, Pfizer, Istanbul) and $10 \mathrm{mg} / \mathrm{kg}$ xylazine (Rompun, Bayer, Istanbul). Following anesthesia, animals were stabilized on the operation table in the prone position. The lumbar region was shaved and cleaned with the antiseptic povidone iodine scrub (MEDICA brush; \%4 chlorhexidine soap, MEDICA BV, Holland) and povidone iodine (POVIOD; \%10 polyvinylpyrrolidone-iodineo complex, Saba, Turkey). The surgical area was closed with sterile drapes. L2-L3 levels were determined. Then, an incision about 1 centimeter $(\mathrm{cm})$ long was made from the midline over the spinous process of the skin.

Distance of the paraspinous muscles was stripped by blunt dissection. Then laminectomy was performed. The rats were divided into three groups:
Control group $(n=6)$ : Only laminectomy was performed at L2L3 levels.

MC group ( $\mathbf{n = 6})$ : MC (0.1 ml, local) was applied where L2-L3 levels laminectomy has done.

ABS group $(\mathbf{n}=6)$ : ABS $(0.1 \mathrm{ml}$, local) was applied where $\mathrm{L} 2-$ L3 levels laminectomy has done.

After 4 weeks, intraperitoneal high dose $(75-100 \mathrm{mg} / \mathrm{kg}$ ) sodium thiopental (Pentothal Sodium Abbott, Italy) was given for euthanasia. The related vertebral column was removed enblock.

The amount of fibrosis and the relationship of fibrosis between dura mater, as well as connective tissue structure in the laminectomy area were evaluated quantitatively by using histological and stereological methods and the results were compared statistically.

\section{Histological Examination}

Each vertebra sample was fixed in $10 \%$ formalin and then decalcified in $30 \%$ formic acid for 2 weeks before embedding. Then, it was dehydrated in a graded alcohol series, and cleared in xylol for light microscopic examination. After dehydration, specimens were embedded in paraffin. Sections were cut using a rotary microtome (Leica, Istanbul, Turkey). Each paraffin block was serially cut at $10 \mu \mathrm{m}$ thickness. The sections were stained with hematoxylin and eosin (H\&E) and Masson's trichrome stain was used for stereological and histopathological examinations. Additionally, immunohistochemical methods were used to determine the mitotic activity. This activity in the related area was evaluated using Ki-67 immune markers.

\section{Stereological Analysis}

Stereological methods provide quantitative descriptions about three-dimensional (3D) structures using two-dimensional (2D) images $(2,12,17)$. The Cavalieri principle is the most commonly used method in stereological volume calculations. The volume of each section obtained from each vertebra sample was estimated by using the Cavalieri method. In the Cavalieri principle, the researcher obtains a data called coefficient of error (CE) to evaluate the reliability of the point density of the grids and sectioning intervals. The CE and coefficient of variation $(\mathrm{CV})$ were estimated according to literature records $(2,17)$.

Sections of $10 \mu \mathrm{m}$ thicknesses with $100 \mu \mathrm{m}$ intervals were acquired. Afterwards, an appropriate point per interested area (total sample areas) carefully calculated for each section.

The volumes of the related samples were estimated with the following formula:

$V=t \times \frac{a}{p} \times \sum_{i=1}^{m} P_{i}$

Where $\mathrm{V}$ is the mean volume of the related area of vertebra, $t$ the mean section thickness, $a / p$ the inter-point area, and $\sum P$ the total number of points hitting whole serial sections of the related area $(12,23)$. 


\section{Statistical Analysis}

Data are presented as $M e a n \pm S E M$, and were statistically analysed. Differences among groups were assessed by ANOVA with the post hoc test to identify individual group differences. Differences were deemed statistically significant at $p<0.05$.

\section{RESULTS}

All stereological results are summarized in Table I.

The total volumes of new bone were estimated by the Cavalieri principle on a series of H\&E stained sections. The estimated volumes of related area belonging to all groups are shown in Figure 1. At this point; the total volume of new bone in the $M C$ group was significantly increased in comparison to the Control group $(p<0.05)$. Besides; there was no significant difference between the other groups.

The total volumes of fibrous tissue were additionally estimated by the Cavalieri principle on a series of H\&E stained sections. The estimated volumes of related area that belong to all groups are shown in Figure 1. There was a highly significant increase in terms of the total volume of fibrous tissue in the $M C$ and ABS groups when compared with the Control group $(p<0.01)$.

All volumetric values of vessels were estimated by the Cavalieri principle on the series of H\&E stained sections. The estimated volumes of related area belonging to all groups are shown in Figure 1 . There was a highly significant difference between the $\mathrm{MC}$ and the Control groups $(\mathrm{p}<0.01)$. Additionally,there was no statistically significant difference $(p>0.05)$ between the other groups.

All stereological findings were suggested by histological (Figures 2A-C, 3A-C) and immunohistochemical (Figures 4A$C, 5 A-C)$ results.

\section{DISCUSSION}

Lumbar laminectomy is one of the most commonly performed spinal surgeries. Unsatisfactory results may occur after laminectomy (14). At this point; PF is an inevitable result of spinal surgery caused by the causative factors in failed back syndrome after spinal surgery (18). There are various studies aiming the prevention of postlaminectomy scarring and

Table I: Total Volume of New Bones, Fibrous Tissues, and Vessels in the Vertebra

\begin{tabular}{lccc}
\hline & \multicolumn{3}{c}{ Groups } \\
\cline { 2 - 4 } Estimation & Control & MC & ABS \\
\hline Total volume of new bone $\left(\mathrm{mm}^{3}\right)$ & $1.6 \pm 0.24$ & $2.09 \pm 0.31$ & $1.5 \pm 0.23$ \\
\hline Total volume of fibrous tissue $\left(\mathrm{mm}^{3}\right)$ & $2.00 \pm 0.22$ & $3.10 \pm 0.3$ & $3.8 \pm 0.35$ \\
\hline Total volume of vessels $\left(\mathrm{mm}^{3}\right)$ & $0.9 \pm 0.16$ & $1.9 \pm 0.46$ & $1.01 \pm 0.18$ \\
\hline
\end{tabular}

Data are presented as Mean \pm SEM.

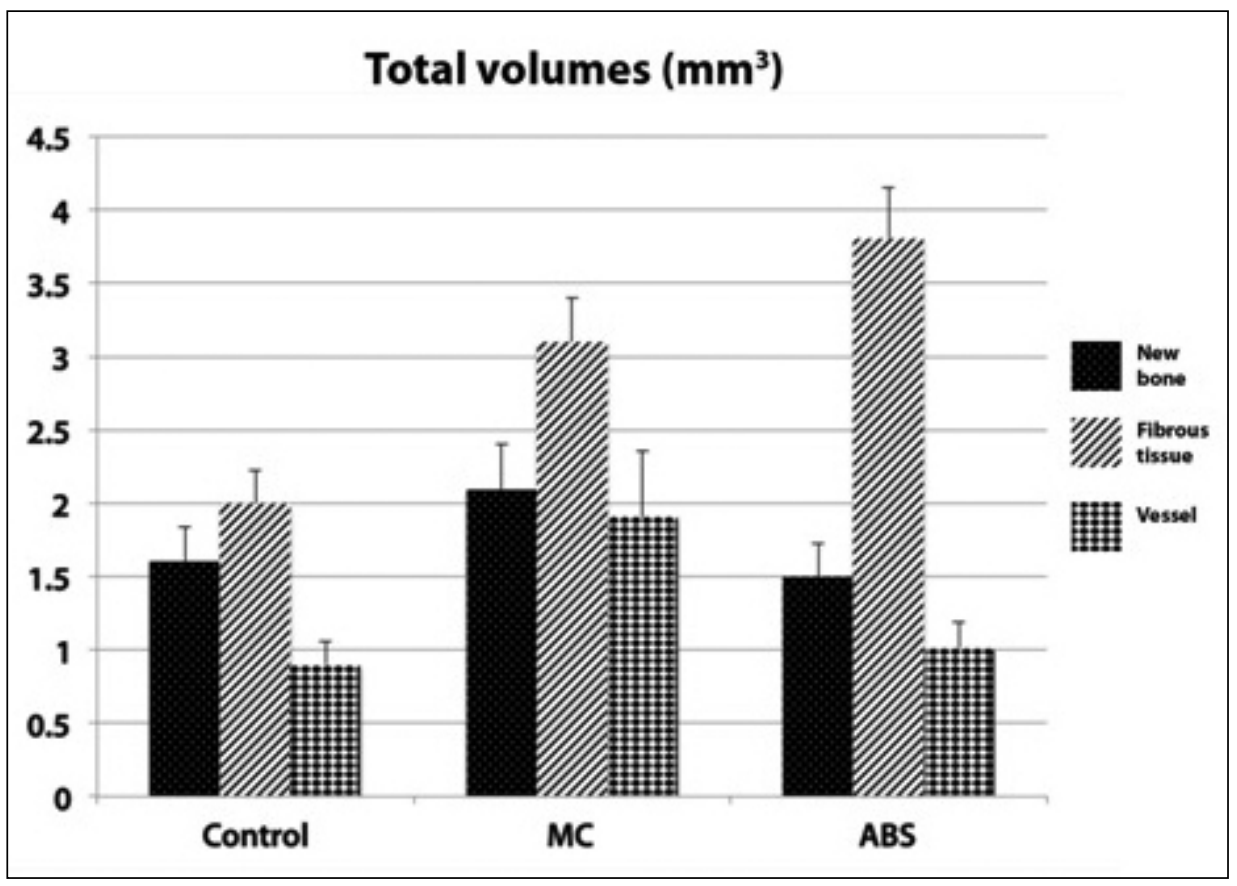

Figure 1: The total volume of new bone, fibrous tissue, and vessels of the groups. There was a significant difference between the MC group and Control group (Mean \pm SEM), $(p<0.05)$ regarding new bone volume. In addition, there was a significant difference also between the ABS and $\mathrm{MC}$ groups in comparison to the Control group in terms of fibrous tissue volume $(p<0.05)$. Regarding the volume of vessel, there was a highly significant increase between the $\mathrm{MC}$ and the Control groups $(\mathrm{p}<0.01)$ whereas no significant difference was found between the Control and ABS groups ( $p>0.05)$. 

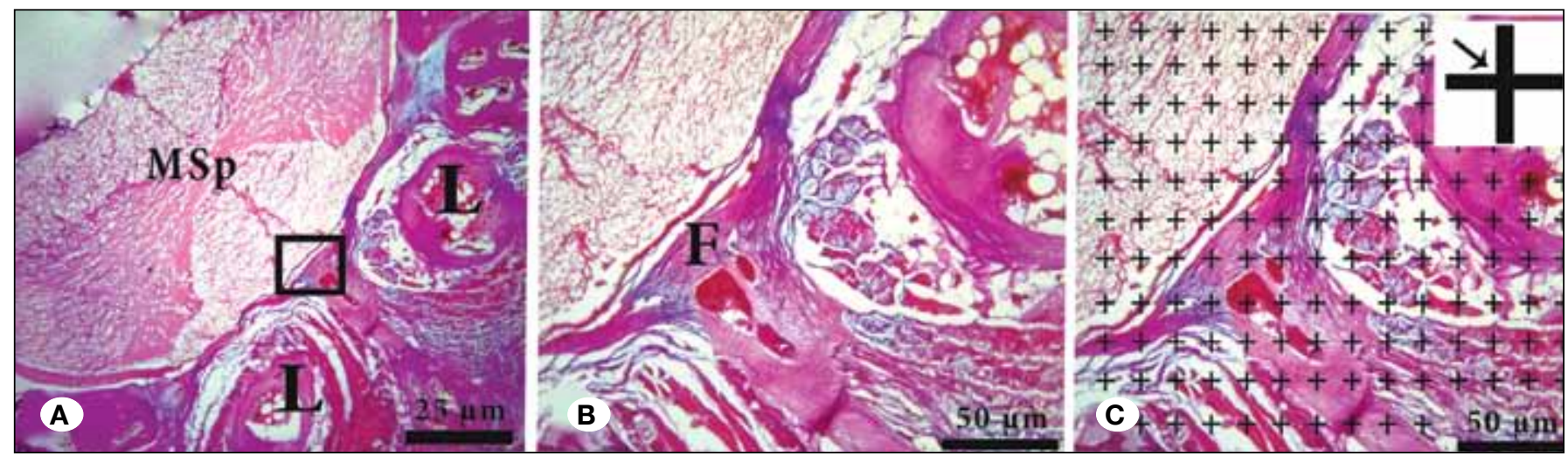

Figure 2: Overview of fibrosis and new bone areas (A, B). Selected sections stained with Masson's trichrome staining method were estimated by using the point counting grid under the light microscope with camera attachment (Zeiss Primostar, Germany). The black arrow indicates the centre of the cross as a point. The number of the points hitting the structure was estimated (C). (MSp: spinal cord; F: fibrous area; L: lamina. Original magnifications: $x 400$ and x1000. Scale bars: $25 \mu \mathrm{m}$ and $50 \mu \mathrm{m})$.
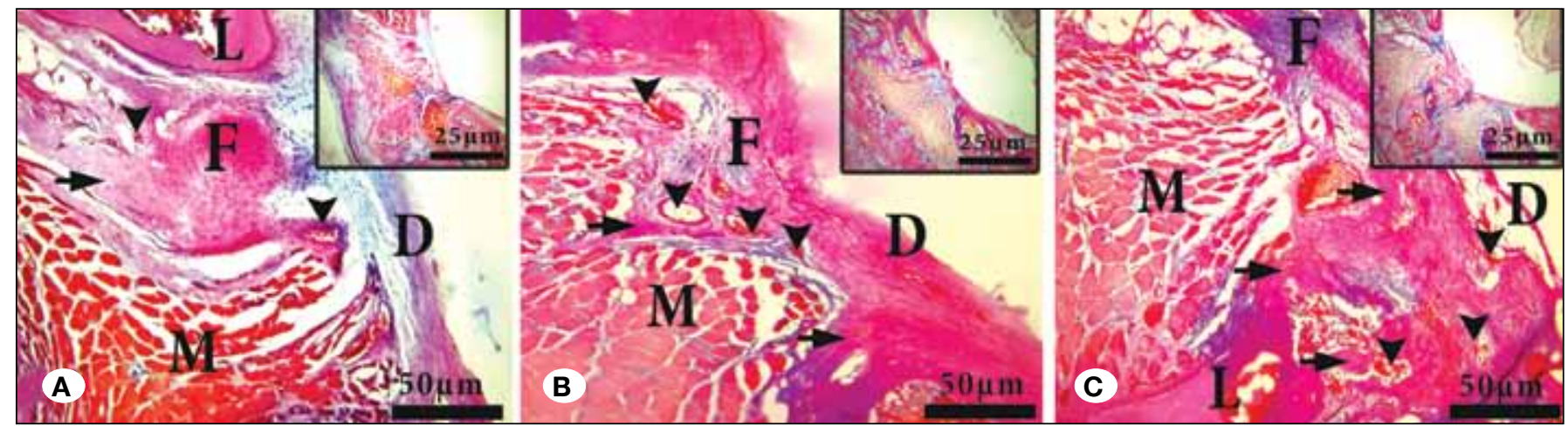

Figure 3: Light microscopic overview of fibrosis and new bone areas after laminectomy. These areas that are indicated by black arrows in the Control group (A), MC group (B), and ABS groups (C) can be seen respectively. Especially the new bone area in MC group, and PF areas between the dura mater and muscle layer in the ABS and Control groups were noted. Arrowheads indicate the vessels. (L: lamina; F: fibrous area; M: muscle layer; D: dura mater. Original magnifications: x400 and x1000. Scale bars: $25 \mu \mathrm{m}$ and $50 \mu \mathrm{m}$. Masson's trichrome staining).
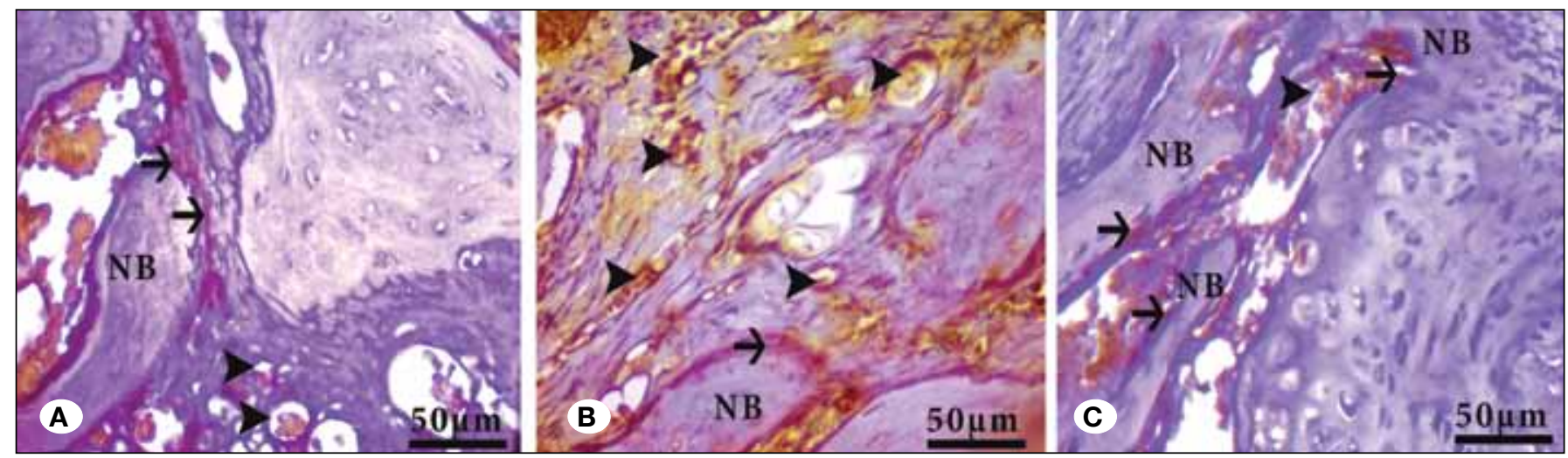

Figure 4: Micrographs of immunohistochemical staining in each group (A-C). Proliferative areas of new bone (black arrow) were shown by $\mathrm{Ki}-67$. Arrowheads indicate proliferation around the vessels. Ki-67 positive cells were stained as brown in the Control group (A), MC group (B), and ABS group (C). It was observed that the proliferative activity was increased in the MC group (B) compared to other groups (NB: new bone area. Scale bars: $50 \mu \mathrm{m}$ ). 

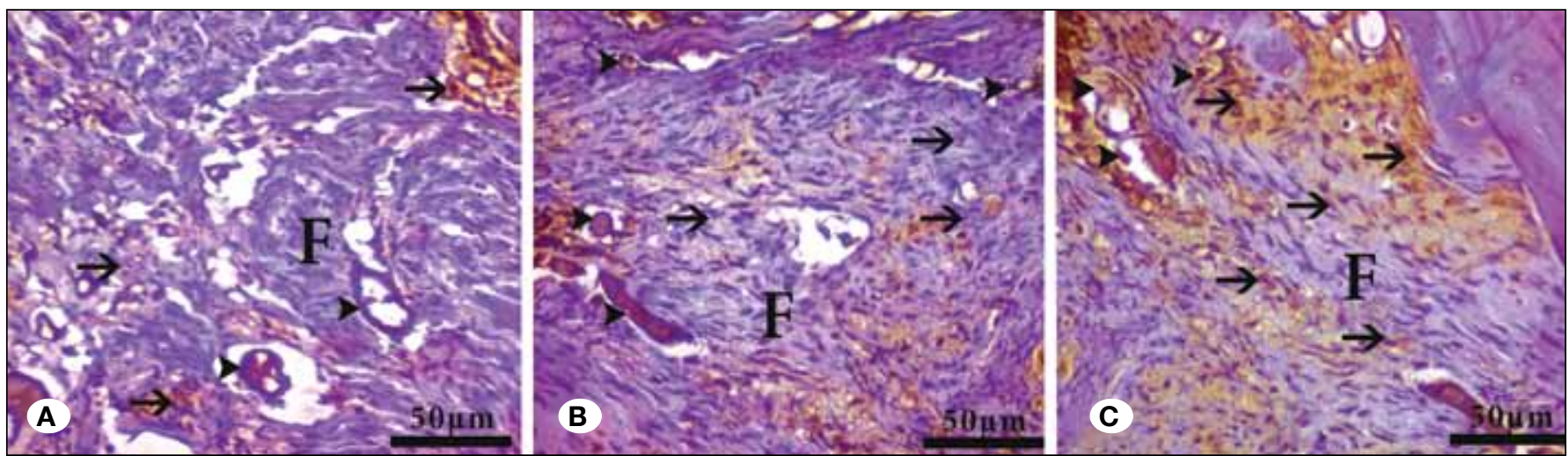

Figure 5: Micrographs of immunohistochemical staining in each group (A-C). Proliferative areas of fibrosis (black arrow) were shown by $\mathrm{Ki}-67$. Arrowheads indicate proliferation around the vessels. Ki-67 positive cells were stained as brown in the Control group (A), ABS group (B), and MC group (C). It was noted that there was increased mitotic activity in fibrous tissues. Increased proliferative activity in the MC group (C) was noted compared to other groups (F: fibrous area. Scale bars: $50 \mu \mathrm{m}$ ).

inducing new bone formation or fusion rate using various mechanical barriers, chemical regulators of scar formation, and prevention of local hematomas $(5,8,30)$.

There is currently no effective medical or surgical treatment for postlaminectomy new bone and PF formations. Reoperation with the intention of excising this fibrous tissue often produces a poor surgical result and further scarring (6). Additionally; many substances have been under investigation to remedy the detrimental effects of postlaminectomy PF. In the literature, we did not find any report investigating the efficiency of $\mathrm{MC}$ and $A B S$ on postoperative new bone and scar formations. In the present study, we aimed to evaluate the effects of $M C$ and ABS on postlaminectomy PF and also new bone formation, and to compare their effects in an experimental laminectomy model.

Analysis of biological tissue samples can be done quantitatively using unbiased stereological techniques if one is dealing with mathematical values such as size of the cells, areas of the related parts, and volume of capillaries in order to validate hypotheses since these approaches are either efficient or reliable $(2,12,17)$.

According to our results, there was significant increase in terms of the total volume of fibrous tissue in the $\mathrm{MC}$ and $\mathrm{ABS}$ groups when compared with the Control group $(p<0.01)$. Because of the antioxidant and wound-healing effects of ABS $(4,25)$, fibrosis, which is a component of wound-healing procedure, was increased in $A B S$ group. Although the osteogenetic effects of ABS seem to be limited, further experimental and clinical studies are required to evaluate its postlaminectomy effects.

The total volumes of new bone and capillaries in the MC group were also significantly increased in comparison to the Control group $(p<0.05)$. MC is a popular medicinal fruit that has been associated with various properties such as anti-microbial, anti-inflammatory and anti-diabetic effects. It has been suggested that its possible effects, which have been mentioned above, are linked to its good source of phenolic compounds and their very high antioxidant activity (28). MC also has an increasing effect on the level of hydroxyproline, which is essential element of collagen synthesis (21). As results of antioxidant properties of $\mathrm{MC}$ by scavenging the free radicals $(1,7)$, and wound healing features by increasing collagen synthesis (21) and enhancing Transforming growth factor- $\beta$ expression (15), it may promote new bone formation and angiogenesis after laminectomy.

\section{CONCLUSION}

Although detailed biochemical analyses and ultrastructural assessments may provide more conclusive results, our results suggested that $M C$ and $A B S$ are not suitable for the prevention of PF in postlaminectomy patients. In addition, $M C$ is especially more convenient for cranial surgery because of its osteogenetic and angiogenetic features. It markedly contributed to bone regeneration.

\section{- REFERENCES}

1. Alam MA, Uddin R, Subhan N, Rahman MM, Jain P, Reza HM: Beneficial role of bitter melon supplementation in obesity and related complications in metabolic syndrome. J Lipids 49:6169, 2015

2. Altunkaynak BZ, Onger ME, Altunkaynak ME, Ayranci E, Canan S: A Brief introduction to stereology and sampling strategies: Basic concepts of stereology. Neuroquantology 10:31-43, 2012

3. Aydincak O, Yilmaz MB, Emmez H, Kurt G, Sepici A, Memis L, Baykaner K: The effect of temozolomide on the prevention of epidural fibrosis developing after lumbar laminectomy in rats. Turk Neurosurg 22:706-711, 2012

4. Bulut E, Bas B, Altunkaynak BZ, Bekcioglu B, Erdem Koc G, Gonulol E, Onger ME, Kaplan S: Efficacy of Ankaferd Blood Stopper on bone healing in diabetic rats: A stereological and histopathological study. Biotech Histochem 89:535-543, 2014

5. Ceviz A, Arslan A, Ak HE, Inaloz S: The effect of urokinase in preventing the formation of epidural fibrosis and/or leptomeningeal arachnoiditis. Surg Neurol 47:124-127, 1997 
6. Circi E, Ozalay M, Caylak B, Bacanli D, Derincek A, Tuncay IC: The effect of oophorectomy on epidural fibrosis after laminectomy: An experimental study in rats. Acta Orthop Traumatol Turc 47:193-200, 2013

7. Duan ZZ, Zhou XL, Li YH, Zhang F, Li FY, Su-Hua Q: Protection of Momordica charantia polysaccharide against intracerebral hemorrhage-induced brain injury through JNK3 signaling pathway. J Recept Signal Transduct Res 35:523-529, 2015

8. Dullerud R, Graver V, Haakonsen M, Haaland AK, Loeb M, Magnaes B: Influence of fibrinolytic factors on scar formation after lumbar discectomy. A magnetic resonance imaging follow-up study with clinical correlation performed 7 years after surgery. Spine (Phila Pa 1976) 23:1464-1469, 1998

9. Farrokhi MR, Vasei M, Fareghbal S, Bakhtazad A: Effect of honey on peridural fibrosis formation after laminectomy in rats: A novel experimental study. Evid Based Complement Alternat Med 50:49-67, 2011

10. Goker H, Haznedaroglu IC, Ercetin S, Kirazli S, Akman U, Ozturk Y, Firat HC: Haemostatic actions of the folkloric medicinal plant extract Ankaferd Blood Stopper. J Int Med Res 36:163-170, 2008

11. Grover JK, Yadav SP: Pharmacological actions and potential uses of Momordica charantia: A review. J Ethnopharmacol 93:123-132, 2004

12. Guney M, Onger ME, Marangoz A, Kaplan S: Surface area estimation: A brief review. Neuroquantology 10:761-764, 2012

13. Gurbuz I, Akyuz C, Yesilada E, Sener B: Anti-ulcerogenic effect of Momordica charantia L. fruits on various ulcer models in rats. J Ethnopharmacol 71:77-82, 2000

14. Gurer B, Kahveci R, Gokce EC, Ozevren H, Turkoglu E, Gokce A: Evaluation of topical application and systemic administration of rosuvastatin in preventing epidural fibrosis in rats. Spine J 15:522-529, 2015

15. Hussan F, Teoh SL, Muhamad N, Mazlan M, Latiff AA: Momordica charantia ointment accelerates diabetic wound healing and enhances transforming growth factor-beta expression. J Wound Care 23:400, 402, 404-407, 2014

16. Jayson MI: The role of vascular damage and fibrosis in the pathogenesis of nerve root damage. Clin Orthop Relat Res:4048, 1992

17. Kaplan S, Odaci E, Canan S, Onger ME, Aslan H, Unal B: The disector counting technique. Neuroquantology 10:44-53, 2012

18. Karatay M, Celik H, Koktekir E, Karatay E, Sertbas I, Yilmaz A, Caydere M, Karabagli $\mathrm{H}$ : Role of tenoxicam in the prevention of postlaminectomy peridural fibrosis in rats. J Neurol Sci 30:559-565, 2013
19. Nagasawa $\mathrm{H}$, Watanabe $\mathrm{K}$, Inatomi $\mathrm{H}$ : Effects of bitter melon (Momordica charantia I.) or ginger rhizome (Zingiber offifinale rosc) on spontaneous mammary tumorigenesis in SHN mice. Am J Chin Med 30:195-205, 2002

20. Ozbakis Dengiz G, Gursan N: Effects of Momordica charantia L. (Cucurbitaceae) on indomethacin-induced ulcer model in rats. Turk J Gastroenterol 16:85-88, 2005

21. Prasad V, Jain V, Girish D, Dorle AK: Wound-healing property of Momordica charantia L. fruit powder. J Herb Pharmacother 6:105-115, 2006

22. Sae-Jung S, Jirarattanaphochai K: Prevention of peridural fibrosis using a cyclooxygenase-2 inhibitor (nonsteroidal antiinflammatory drug) soaked in absorbable gelatin sponge: An experimental comparative animal model. Spine (Phila $\mathrm{Pa}$ 1976) 38:E985-991, 2013

23. Sahin B, Elfaki A: Estimation of the volume and volume fraction of brain and brain structures on radiological images. Neuroquantology 10:87-97, 2012

24. Sandoval MA, Hernandez-Vaquero D: Preventing peridural fibrosis with nonsteroidal anti-inflammatory drugs. Eur Spine J 17:451-455, 2008

25. Sarikas NG, Korkmaz T, Kahramansoy N, Kilicgun A, Boran C, Boztas G: The effects of ankaferd blood stopper on the recovery process in an experimental oesophageal perforation model. Balkan Med J 32:96-100, 2015

26. Sathishsekar D, Subramanian S: Beneficial effects of Momordica charantia seeds in the treatment of STZ-induced diabetes in experimental rats. Biol Pharm Bull 28:978-983, 2005

27. Simsek HO, Tuzum MS, Baykul T, Gurer IE, Bassorgun Cl: Experimental investigation of the effects of a blood stopper agent (ankaferd blood stopper) on bone surfaces. Turk $\mathrm{J}$ Haematol 30:177-183, 2013

28. Tan SP, Stathopoulos C, Parks S, Roach P: An optimised aqueous extract of phenolic compounds from Bitter Melon with high Antioxidant capacity. Antioxidants (Basel) 3:814829, 2014

29. Wang HL, Boyapati L: "PASS" principles for predictable bone regeneration. Implant Dent 15:8-17, 2006

30. Yucesoy K, Karci A, Kilicalp A, Mertol T: The barrier effect of laminae: Laminotomy versus laminectomy. Spinal Cord $38: 442-444,2000$

31. Zheng ZX, Teng JY, Liu JY, Qiu JH, Ouyang H, Xue C: The hypoglycemic effects of crude polysaccharides extract from Momordica charantia in mice. Wei Sheng Yan Jiu 34:361-363, 2005 